arising through the same pathway and predisposing to adenocarcinoma development at this site. The influence of obesity on the aetiology of cardia mucosa is unknown.

Methods $62 \mathrm{H}$ pylori negative healthy volunteers (age 18-74 years) were recruited. BMI, waist circumference and gender were recorded. MRI (Phillips 1.5T) was performed for quantification of visceral and subcutaneous fat (average of three axial planes; L2, L3 and L4). Upper GI endoscopy was performed with biopsies of the gastrooesophageal junction. Biopsies were taken in a craniocaudal direction and targeted to include enough squamous mucosa to confirm position. Intra-procedure pathological feedback was available and two to three biopsies were taken to optimise accuracy. Junctional biopsies were assessed to determine cardia length, considered measurable provided there was consecutive squamous, cardia and oxyntic mucosal types present. Non-parametric correlations were examined between BMI, waist circumference and cardia length and between fat distribution quantified by MRI and cardia length. Regression analysis (Stepwise method) incorporating age, BMI, waist circumference and MRI total fat was used to determine predictors of cardia length.

Results 37 of 62 volunteers had at least one junctional biopsy including squamous, cardia and oxyntic mucosa; median total length $6.5 \mathrm{~mm}$ (IOR 1.6). Median cardia mucosal length was $2.5 \mathrm{~mm}$ (IOR $1.5 \mathrm{~mm}$ ). Length of cardia mucosa increased with age $(R=0.457, p=0.004)$ and with waist circumference $(R=0.466$, $\mathrm{p}=0.004)$. A correlation was also seen with intra-abdominal fat $(R=0.374, p=0.027)$ and total fat measured by MRI $(R=0.389$, $p=0.021)$ but not with subcutaneous fat $(p=0.091)$. There was no significant correlation with BMI. On regression analysis the independent predictors of cardia mucosa length were waist circumference (Standardised coefficient 0.342, $\mathrm{p}=0.035$ ) and age (Standardised coefficient $0.322, \mathrm{p}=0.046$ ). Intestinal metaplasia at the cardia was seen in only 4 of 62 volunteers.

Conclusion These findings suggest that cardia mucosa may be acquired with increasing age through a process of distal squamous columnar metaplasia accelerated by central obesity. A possible mechanism is opening of the distal portion of the lower oesophageal sphincter and short segment acid reflux.

Competing interests None declared.

\section{PTU-177 REDUCTION IN LENGTH OF STAY FOR PATIENTS UNDERGOING MAJOR UPPER GASTROINTESTINAL RESECTIONS WITH IMPLEMENTATION OF ENHANCED RECOVERY PACKAGES}

doi:10.1136/gutjnl-2012-302514c.177

${ }^{1}$ E Gemmill, ${ }^{*}{ }^{1} \mathrm{~J}$ Tang, ${ }^{2} \mathrm{D}$ Humes, ${ }^{3} \mathrm{P}$ Daliya, ${ }^{1} \mathrm{~N}$ Welch, ${ }^{1} \mathrm{~S}$ Parsons, ${ }^{1} \mathrm{~J}$ Catton. ${ }^{1} \mathrm{UGl}$ Surgery, Nottingham City Hospital, Nottingham, UK; ${ }^{2}$ Nottingham Digestive Disease Centre and Biomedical Research Unit, Nottingham, UK: ${ }^{3}$ General Surgery, Nottingham City Hospital, Nottingham, UK

Introduction The high mortality and morbidity associated with resection for gastro-oesophageal malignancy has resulted in a conservative approach to the post-operative management of this patient group. In August 2009 an Enhanced Recovery After Surgery (ERAS) pathway tailored for patients undergoing resection for gastro-oesophageal malignancy was introduced. We aimed to assess the impact of this change in practice on standard clinical outcomes. Methods We performed a retrospective review of two cohorts of patients undergoing resection for gastro-oesophageal malignancy before (08/08-07/09) and after (08/09-07/10) the implementation of the ERAS pathway. Data were collected on demographics, interventions, length of stay, morbidity and in hospital mortality.

Results There were 53 and 55 gastro-oesophageal resections undertaken in each year for malignant disease. The median length of stay for both gastric and oesophageal resection decreased from 15 to 11 days $(p<0.001)$ following the implementation of the ERAS pathway. There was no increase in morbidity (gastric resection $23.1 \%$ vs $5.3 \%$ and oesophageal resection $25.9 \%$ vs $16.7 \%$ ) or mortality (gastric resection no deaths and oesophageal resection $1.8 \%$ vs $3.6 \%$ ) associated with the changes. There was a significant decrease in the number of oral contrast studies used following oesophageal resection with a reduction from $21(77.8 \%)$ in $2008-2009$ to $6(16.7 \%)$ in $2009-2010\left(\chi^{2}, \mathrm{p}<0.0001\right)$.

Conclusion The introduction of an enhanced recovery programme following gastro-oesophageal surgery resulted in a significant decrease in length of median patient stay in hospital without a significant increase in associated morbidity and mortality.

Competing interests None declared.

\section{PTU-178 MEASUREMENT OF ESOPHAGO-GASTRIC JUNCTION CROSS-SECTIONAL AREA AND DISTENSIBILITY BY ENDOFLIP ${ }^{\circledR}$ (ENDOLUMENAL FUNCTIONAL LUMEN IMAGING PROBE) FOR THE DIAGNOSIS OF PATIENTS WITH GASTRO-ESOPHAGEAL REFLUX DISEASE (GERD)}

doi:10.1136/gutjnl-2012-302514c.178

${ }^{1} \mathrm{E}$ Tucker, ${ }^{*} \mathrm{R}$ Sweis, ${ }^{2} \mathrm{~A}$ Anggiansah, ${ }^{1} \mathrm{E}$ Telakis, ${ }^{3} \mathrm{~J}$ Wright, ${ }^{4} \mathrm{~K}$ Knowles, ${ }^{2} \mathrm{~T}$ Wong, ${ }^{1} \mathrm{M}$ Fox. ${ }^{1} \mathrm{NIHR}$ Biomedical Research Unit, University of Nottingham, Nottingham, UK; ${ }^{2}$ Oesophageal Laboratory, Guys and St Thomas's NHS Foundation Trust, London, UK; ${ }^{3}$ Division of GI Surgery, University of Nottingham, Nottingham, UK; ${ }^{4}$ Motility Unit, Nottingham University Hospital Trust, Nottingham, UK

Introduction EndoFLIP ${ }^{\circledR}$ (Crospon, Ireland) is an innovative device designed to assess the cross sectional area (CSA) and distensiblilty of the esophagogastric junction (EGJ) by combined impedance planimetry and pressure measurement. Initial studies have suggested that this probe may distinguish between gastro-oesophageal reflux disease (GERD) patients and healthy volunteers (HV).

Aim To assess the diagnostic agreement of EndoFLIP ${ }^{\circledR}$ measurements with clinical and physiologic GERD diagnosis.

Methods 22 healthy volunteers, (HV; female $=16$, age $21-46$, mean body mass index (BMI) $24.3 \mathrm{~kg} / \mathrm{m}^{2}$ ) and 20 patients with GERD symptoms (female $=14$, age $19-78$, mean BMI $33.2 \mathrm{~kg} / \mathrm{m}^{2}$ ) were studied. Patients were older $(p<0.0001)$ and had greater BMI $(p=0.001)$. Median EGJ CSA and distensibility at $20 \mathrm{ml}$ and $30 \mathrm{ml}$ EndoFLIP ${ }^{\circledR}$ balloon volume were measured. A Bravo capsule (Given Imaging, Israel) was attached $6 \mathrm{~cm}$ above the Z-line and a $48 \mathrm{~h}$ wireless esophageal $\mathrm{pH}$ recording acquired. The ability of EndoFLIP ${ }^{\circledR}$ measurements to discriminate (1) patient group and (2) individuals with pathologic acid exposure $(>5.6 \%$ time $<\mathrm{pH} 4)$ was calculated. Results Complete measurements were acquired except in one patient with early detachment of Bravo capsule. Distensibility could not be measured in one patient and one volunteer with negative endoFLIP ${ }^{\circ}$ balloon pressures. $7 / 22$ (32\%) HVs and 7/19 (37\%) of patients had oesophagitis (six patients with hiatus hernia). 3/22 (14\%) HVs and 9/19 (47\%) patients had pathologic acid exposure $(p=0.126)$. EGJ CSA was higher in healthy volunteers than the patient group, at $20 \mathrm{ml}(p=0.018)$ and $30 \mathrm{ml}(p=0.058$, Abstract PTU-178 figure 1) endoFLIP ${ }^{\circledR}$ balloon volume. EGJ distensibility was lower in patients than HVs at $20 \mathrm{ml}(\mathrm{p}=0.001)$ and $30 \mathrm{ml}$ balloon volume, $(p=0.020$, Abstract PTU-178 figure 1). EndoFLIP ${ }$ measurements were similar in participants with and without pathologic acid exposure (median CSA $40 \mathrm{~mm}^{2}$ vs $34 \mathrm{~mm}^{2} \mathrm{p}=1.0$ at $20 \mathrm{ml}, 98 \mathrm{~mm}^{2}$ vs $107 \mathrm{~mm}^{2}, \mathrm{p}=0.789$ at $30 \mathrm{ml}$ ) and distensibility at $20 \mathrm{ml}(\mathrm{p}=0.574)$ and $30 \mathrm{ml}$ balloon volume $(\mathrm{p}=0.704)$. Post-hoc analysis revealed an inverse association between BMI and CSA $\left(R^{2}=0.214, p=0.003\right)$ and negative association with distensibility $\left(\mathrm{R}^{2}=0.211, \mathrm{p}=0.003\right)$. BMI was associated also with a trend to increased acid exposure $(p=0.116)$. 